\title{
Gene expression profile and enrichment pathways in different stages of bladder cancer
}

\author{
Z.-Q. Fang', W.-D. Zang ${ }^{2}$, R. Chen ${ }^{3}$, B.-W. Ye ${ }^{1}$, X.-W. Wang', S.-H. Yi', \\ W. Chen ${ }^{1}$, F. He ${ }^{1}$ and G. Ye ${ }^{1}$ \\ ${ }^{1}$ Department of Urology, Center of Nephrology, \\ The Second Affiliated Hospital, The Third Military Medical University, \\ Chongqing, China \\ ${ }^{2}$ Bio-X Center, Key Laboratory for the Genetics of Developmental and \\ Neuropsychiatric Disorders, Shanghai Jiao Tong University, \\ Ministry of Education, Shanghai, China \\ ${ }^{3}$ Department of Pathology, Center of Nephrology, \\ The Second Affiliated Hospital, The Third Military Medical University, \\ Chongqing, China \\ Corresponding author: G. Ye \\ E-mail: gangyegy@hotmail.com
}

Genet. Mol. Res. 12 (2): 1479-1489 (2013)

Received May 28, 2012

Accepted January 19, 2013

Published May 6, 2013

DOI http://dx.doi.org/10.4238/2013.May.6.1

\begin{abstract}
Bladder cancer is a highly heterogeneous neoplasm. We examined the gene expression profile in 3 bladder cancer stages (Ta, T1, T2) using expression microarray analysis of 40 bladder tumors. Differentially expressed genes were found by the $t$-test, with $<0.005$ as the significance threshold. KEGG pathway-enrichment analysis was used to study the signaling pathways of the genes. We found 36 genes that could be used as molecular markers for predicting the transition from Ta-T1 to T1-T2. Among these, 11 overlapped between Ta-T1 and T1-T2 stages. Six genes were downregulated at the Ta-T1 stage, but were up-regulated at the T1-T2 stage ( $A N X A 5$, $A T P 6 V 1 B 2, C T G F, G E M$, IL13RA1, and LCP1); 5 genes were up-regulated at the Ta-T1 stage, but down-regulated at the T1-T2 stage (ACPP, GNL1, RIPK1, $R A P G E F 3$, and $Z E R I)$. Another 25 genes changed relative expression levels at the T1-T2 stage. These genes (including COL1A1, COL1A2, FN1, ITGA5,
\end{abstract}


LGALS1, SPP1, VIM, POSTN, and COL18A1) may be involved in bladder cancer progression by affecting extracellular matrix-receptor interaction and focal adhesion. The cytokine-cytokine receptor interaction, neuroactive ligandreceptor interaction, and calcium-signaling pathway were associated with bladder cancer progression at both the Ta-T1 and T1-T2 stages.

Key words: Bladder cancer; KEGG pathway; Tumor grades; Ta-T1; T1-T2

\section{INTRODUCTION}

Bladder cancer is the most common malignancy of the urinary tract. The incidence rate is 10.1 per 100 men and 2.5 per 100 women (Ramírez-Backhaus et al., 2012). Bladder cancer consists of highly heterogeneous tumors. Approximately $70-80 \%$ of bladder tumors are superficial. Among them, $70 \%$ present as Ta lesions located only in the mucosa, 20\% present as $\mathrm{T} 1$ lesions located in the submucosa, and only $10 \%$ present as carcinoma in situ lesions (Kirkali et al., 2005). While patients belonging to the first 2 groups have a high risk of recurrence after treatment (50\% for Ta tumors; $70 \%$ for T1 tumors), but a low disease progression rate (10\% for Ta tumors; $30 \%$ for T1 tumors), patients diagnosed with concomitant carcinoma in situ lesions have an increased risk of disease progression to a muscle invasive stage. The remaining $20-30 \%$ of bladder tumors show muscle infiltration at the time of initial presentation (T2-4). Half of these patients already harbor or will eventually develop distant metastases (Kirkali et al., 2005). Therefore, it is important to accurately predict the course of disease and to provide appropriate treatment regimens for patients.

Recent studies have revealed that superficial or invasive bladder cancers are associated with distinct genotypic and phenotypic patterns. Superficial tumors are characterized by gainof-function mutations affecting oncogenes such as HRAS (Burchill et al., 1994), FGFR3 (van Rhijn et al., 2001), and PIK3CA (López-Knowles et al., 2006). In addition, candidate suppressor genes mapping to the long arm of chromosome 9, such as TSC1 (Hornigold et al., 1999), PTCH (McGarvey et al., 1998), DBCl (Louhelainen et al., 2006), and INK4A (Orlow et al., 1999), also appear to be involved, at least in the initiation of certain papillary non-invasive neoplasms. In contrast, invasive tumors are associated with loss-of-function mutations, affecting tumor suppressor genes such as Tp53, RB (Castillo-Martin et al., 2010), and PTEN (Han et al., 2008). Some oncogenes, including HDM2 (Sanchez-Carbayo et al., 2007), E2F genes (e.g., E2F1 and E2F3), and $A K T$ (Askham et al., 2010), which affect the P53, RB, and PTEN pathways, respectively, have also been reported to be involved in the progression of these neoplasms.

Numerous biological markers have been identified and correlated to some extent with tumor stage. However, the power of many of these markers in predicting the clinical outcomes of individual tumors is limited because of low specificity. Therefore, alternative markers are still needed for the detection of the disease and for predictive purposes. One potential approach to identify such markers involves the molecular profiling of tumors at different stages by high-throughput technologies such as microarray analysis of gene expression. In this study, we aimed to use microarray data to identify specific molecular markers of the various stages of bladder cancer. The Kyoto Encyclopedia of Genes and Genomes (KEGG) pathway-enrichment analysis was also performed to explore the signaling pathways in which the differentially expressed genes may be involved. We anticipate that these studies will provide a more comprehensive understanding of bladder tumor stage transition. 


\section{MATERIAL AND METHODS}

\section{Affymetrix microarray data}

Affymetrix bladder tumor microarray datasets were obtained from the National Center for Biotechnology Information (NCBI) Gene Expression Omnibus data repository (http:// www.ncbi.nlm.nih.gov/geo/) using the series accession number GSE89. The tissue samples used for microarray analysis were classified into 3 categories: Ta with superficial growth (papillary tumor limited to mucosa, without penetration into the lamina propria), $\mathrm{T} 1$ or intermediary growth (with extension into but not beyond lamina propria), and T2 or invasive growth (tumor with invasion into superficial muscle layers). The 40 tissue samples used for the microarray study consisted of $20 \mathrm{Ta}, 11 \mathrm{~T} 1$, and $9 \mathrm{~T} 2$ tumors.

\section{Array normalization and statistical analysis of differentially expressed genes (DEGs)}

Raw gene expression data were normalized using the Robust Multiarray Average method implemented in the Affymetrix package. The significance of gene expression differences for the 3 pairwise comparisons made (Ta-T1, Ta-T2, and T1-T2) was tested by the $t$-test and the $\mathrm{P}$ values were adjusted for multiple comparisons using the false-discovery rate (FDR) of Benjamini and Hochberg (1995). FDR-corrected P values $<0.05$ were considered to be statistically significant.

\section{Protein-protein interaction (PPI) data}

The Human Protein Reference Database (HPRD) is a protein database accessible through the internet (http://www.hprd.org/). PPI data were collected from the HPRD database, which provides 37,041 interactions for 9,518 proteins.

\section{KEGG pathway-enrichment analysis}

The KEGG (http://www.genome.jp/kegg/) is a collection of online databases of genomes, enzymatic pathways, and biological chemicals. The 'pathway' database, which contains approximately 300 biological pathways, records the networks of molecular interactions in cells as well as their variants specific to particular organisms. SubpathwayMiner (Li et al., 2009) was used to convert metabolic pathways to enzyme-enzyme (KEGG Orthology, KO$\mathrm{KO})$ pathway graphs, with enzymes as nodes and compounds as edges. The K-Cliques method was used to identify sub-pathways based on pathway structure data provided by KEGG, with the $\mathrm{K}$ value setting as 3 . FDR $<0.005$ was considered as the significance threshold.

\section{RESULTS}

\section{DEGs in the Ta-T1, Ta-T2, and T1-T2 transitions}

DEGs at the 3 transitions (Ta-T1, Ta-T2 and T1-T2) were identified by the $t$-test using an FDR-corrected P value $<0.05$ as the significance threshold. We identified 2055 DEGs 
at the Ta-T1 transition, of which 940 genes (45.7\%) were down-regulated and 1115 genes $(54.3 \%)$ were up-regulated. In the Ta-T2 comparison, 1876 DEGs were identified, of which 934 genes (49.8\%) were down-regulated and 942 genes (50.2\%) were up-regulated. Only 38 DEGs were identified at the T1-T2 transition, of which 12 genes $(31.6 \%)$ were downregulated and 26 genes $(68.4 \%)$ were up-regulated.

Venn diagrams were used to display the DEGs that overlapped between the Ta-T1, Ta-T2, and T1-T2 comparisons (Figure 1). We found that 13 genes overlapped between the $\mathrm{Ta}-\mathrm{T} 1$ and T1-T2 transitions. However, 11 of these genes showed expression changes in opposite directions at the 2 transitions. Six genes were down-regulated at the Ta-T1 transitions, but up-regulated at the T1-T2 transition. On the contrary, 5 genes were up-regulated at the Ta-T1 transition, but down-regulated at the T1-T2 transition (Table 1). Only CCNG2 and CLCN3 were both down-regulated at both transitions. Similarly, 21 DEGs were common to the Ta-T2 and T1-T2 transitions. However, the direction of expression change of these 21 genes was similar at both transitions, with 5 genes showing down-regulation and 16 showing up-regulation (Table 2). Importantly, 1202 DEGs were common to the Ta-T1 and Ta-T2 comparisons, of which, the expression profiles of 2 genes (CCNG2 and $C L C N 3$ ) changed in all 3 comparisons (Figure 1).

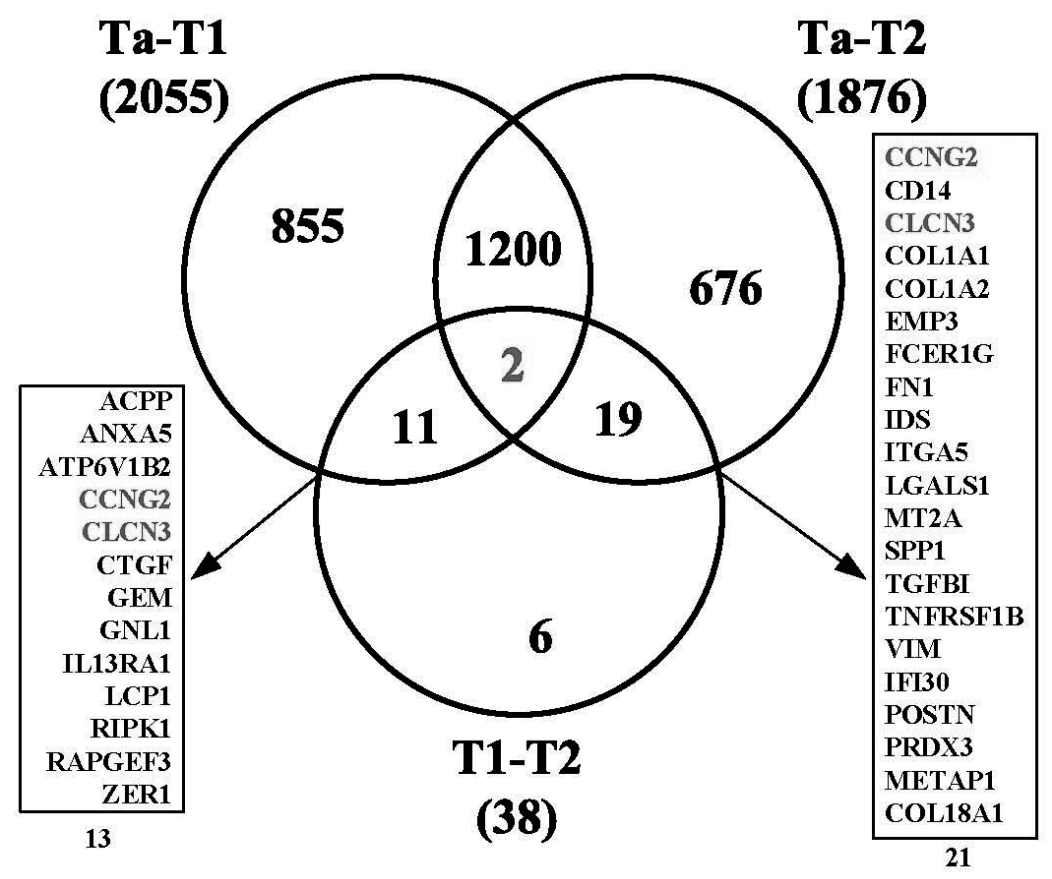

Figure 1. Venn diagram showing overlap of genes differentially expressed in Ta-T1, Ta-T2, and T1-T2.

\section{PPI network and pathway-enrichment analysis}

Because the expression of 1200 genes was found to be dysregulated (up-regulated or down-regulated) at the Ta-T1 and Ta-T2 transitions, we attempted to investigate their 
potential biological functions by constructing a PPI network and by performing a pathwayenrichment analysis. The results indicated that the PPI network with the largest connected component included 487 nodes and 792 edges, and that $60 \%$ of the genes (290) within this component were in the KEGG pathway database (Figure 2). Furthermore, we analyzed 4 topological properties - degree, betweenness, closeness, and cluster coefficient - of the 487 genes whose expression was dysregulated in all 3 comparisons as well as other genes included in the PPI network (Figure 3). We found that the 4 topological property values of the genes whose expression was dysregulated in all 3 comparisons were higher than that of all genes in the PPI network. This suggests that these dysregulated genes are located at an important topological site in the PPI network, and thus, may play an important role in bladder cancer development.

Table 1. Changes in expression of 13 genes at Ta-T1 and T1-T2 stages.

\begin{tabular}{llllc}
\hline Gene ID & Symbol & Ta-T1 & T1-T2 & Same direction \\
\hline 901 & $C C N G 2$ & Down & Down & Yes \\
1182 & $C L C N 3$ & Down & Down & Yes \\
308 & $A N X A 5$ & Down & Nown & No \\
526 & $A T P 6 V 1 B 2$ & Down & Up & No \\
1490 & $C T G F$ & Down & Up & No \\
2669 & GEM & Down & Up & No \\
3597 & $I L 13 R A 1$ & Down & Up & No \\
3936 & $L C P 1$ & $\mathrm{Up}$ & Up & No \\
55 & $A C P P$ & $\mathrm{Up}$ & Down & No \\
2794 & GNL1 & $\mathrm{Up}$ & Down & No \\
8737 & $R I P K 1$ & $\mathrm{Up}$ & Down & No \\
10411 & $R A P G E F 3$ & $\mathrm{Up}$ & Down & \\
10444 & ZER1 & & & \\
\hline
\end{tabular}

Table 2. Changes in expression of 21 genes at Ta-T2 and T1-T2 stages.

\begin{tabular}{|c|c|c|c|c|}
\hline Gene ID & Symbol & Ta-T2 & $\mathrm{T} 1-\mathrm{T} 2$ & Same direction \\
\hline 901 & $C C N G 2$ & Down & Down & Yes \\
\hline 1182 & $C L C N 3$ & Down & Down & Yes \\
\hline 3423 & $I D S$ & Down & Down & Yes \\
\hline 10935 & $P R D X 3$ & Down & Down & Yes \\
\hline 23173 & METAPI & Down & Down & Yes \\
\hline 929 & $C D 14$ & Up & $\mathrm{Up}$ & Yes \\
\hline 1277 & COL1A1 & Up & Up & Yes \\
\hline 1278 & COL1A2 & Up & Up & Yes \\
\hline 2014 & EMP3 & Up & Up & Yes \\
\hline 2207 & FCERIG & Up & Up & Yes \\
\hline 2335 & FN1 & Up & $\mathrm{Up}$ & Yes \\
\hline 3678 & ITGA5 & Up & Up & Yes \\
\hline 3956 & LGALSI & $\mathrm{Up}$ & $\mathrm{Up}$ & Yes \\
\hline 4502 & $M T 2 A$ & Up & $\mathrm{Up}$ & Yes \\
\hline 6696 & $S P P 1$ & Up & Up & Yes \\
\hline 7045 & $T G F B I$ & Up & $\mathrm{Up}$ & Yes \\
\hline 7133 & TNFRSFIB & $\mathrm{Up}$ & $\mathrm{Up}$ & Yes \\
\hline 7431 & $V I M$ & $\mathrm{Up}$ & $\mathrm{Up}$ & Yes \\
\hline 10437 & IFI30 & Up & Up & Yes \\
\hline 10631 & POSTN & Up & Up & Yes \\
\hline 80781 & COL18A1 & Up & Up & Yes \\
\hline
\end{tabular}




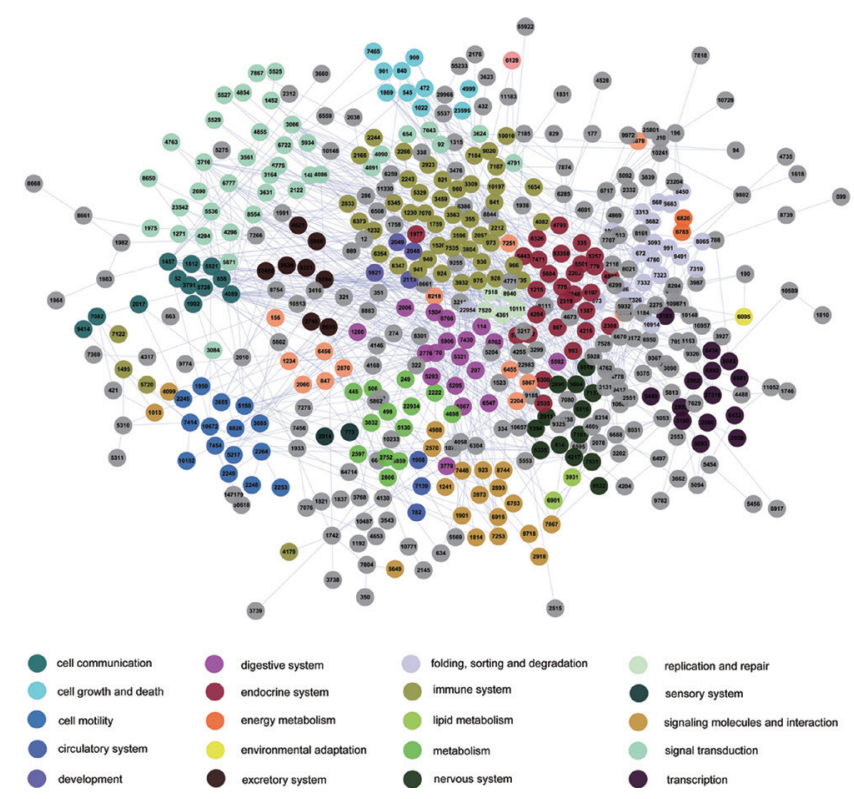

Figure 2. Protein-protein interaction network and matched biological pathway at Ta, T1, and T2 stages. The different colors indicate the matched biological pathway for these genes. The gray node indicates that genes were not annotated into matched biological pathway.
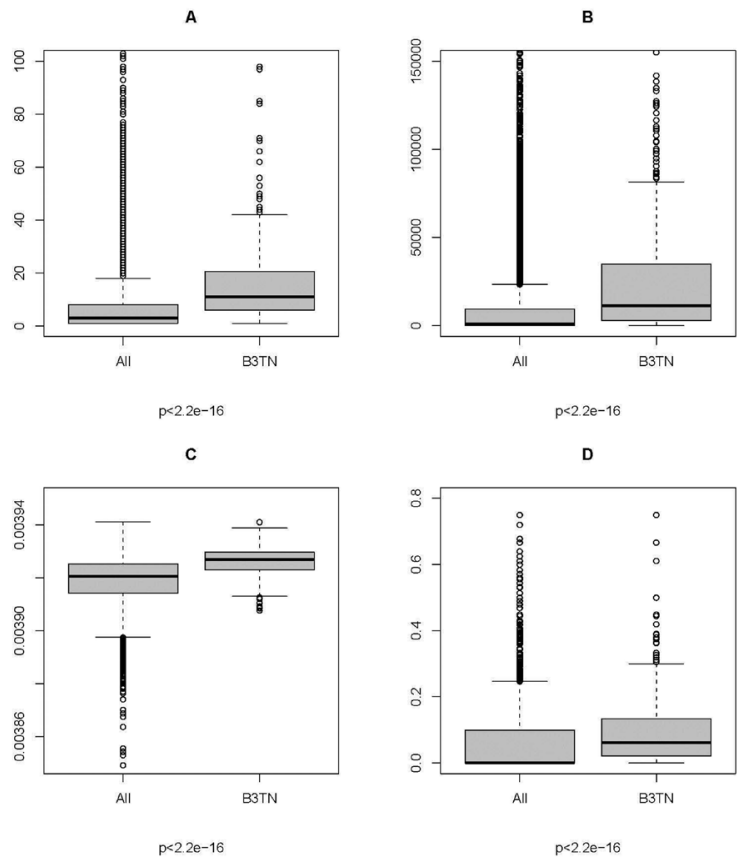

Figure 3. Four topological properties of bladder cancer-related genes at 3 stages in the protein-protein interaction (PPI) network. A. Degree; B. betweenness; C. closeness; D. cluster coefficient. In these 4 topological properties, the expression of bladder cancer-related genes at 3 stages was higher than that of other genes in the PPI network. 
Through the pathway-enrichment analysis, 43 pathways, including cytokine-cytokine receptor interaction, neuroactive ligand-receptor interaction, the calcium-signaling pathway, the insulin-signaling pathway, the MAPK-signaling pathway, and the JAK-STAT-signaling pathway, among others (Table 3, only the top 10 pathways are listed), were found to play an important role at various stages of bladder cancer.

\begin{tabular}{l}
\multicolumn{3}{c}{ Table 3. KEGG pathway-enrichment analysis (the top 10 pathways) for 1200 differentially expressed genes. } \\
\begin{tabular}{llcc}
\hline Pathway ID & Pathway name & P value & FDR \\
\hline path:04060 & Cytokine-cytokine receptor interaction & $1.20 \mathrm{E}-10$ & $3.56 \mathrm{E}-09$ \\
path:04080 & Neuroactive ligand-receptor interaction & $2.53 \mathrm{E}-12$ & $3.56 \mathrm{E}-09$ \\
path:04020 & Calcium-signaling pathway & $1.46 \mathrm{E}-10$ & $1.23 \mathrm{E}-08$ \\
path:04910 & Insulin-signaling pathway & $1.17 \mathrm{E}-09$ & $4.23 \mathrm{E}-08$ \\
path:04010 & MAPK-signaling pathway & $2.26 \mathrm{E}-08$ & $1.02 \mathrm{E}-06$ \\
path:04630 & JAK-STAT-signaling pathway & $1.45 \mathrm{E}-09$ & $1.02 \mathrm{E}-06$ \\
path:04062 & Chemokine-signaling pathway & $1.27 \mathrm{E}-07$ & $3.82 \mathrm{E}-06$ \\
path:04720 & Long-term potentiation & $2.88 \mathrm{E}-08$ & $3.82 \mathrm{E}-06$ \\
path:04810 & Regulation of actin cytoskeleton & $1.85 \mathrm{E}-07$ & $3.82 \mathrm{E}-06$ \\
path:04260 & Cardiac muscle contraction & $5.90 \mathrm{E}-06$ & $7.58 \mathrm{E}-05$ \\
\hline
\end{tabular}
\end{tabular}

$\mathrm{FDR}=$ false-discovery rate

Additionally, of the 38 genes identified as DEGs at the T1-T2 transition, 13 genes also showed differential expression at the Ta-T1 transition, whereas 25 genes showed differential expression exclusively at the T1-T2 transition. Therefore, we also performed the functional annotation of these 25 genes and found them to be involved in 2 important pathways: extracellular matrix (ECM)-receptor interaction and focal adhesion.

\section{DISCUSSION}

In this study, we aimed to investigate the changes in gene expression at the different stages of bladder cancer. We found the expression of 11 genes to be dysregulated in both the Ta-T1 and T1-T2 transitions, although these changes occurred in opposite directions in the 2 comparisons. Six genes were down-regulated at the Ta-T1 transition, but up-regulated at the T1-T2 transition (ANXA5, ATP6V1B2, CTGF, GEM, IL13RA1, and LCP1). On the other hand, 5 genes were up-regulated at the Ta-T1 transition but down-regulated at the T1-T2 stage (ACPP, GNL1, RIPK1, RAPGEF3, and ZER1). These results suggest that the expression changes in these 11 genes may play an important role in the transition from the Ta to T1 stage and from the T1 to T2 stage. These expression changes are in accordance with previous studies as the following described.

ANXA5 (Annexin 5) is used as a genetic marker of apoptosis and has been shown to influence susceptibility to apoptosis and the pro-inflammatory activities of apoptotic cells. Alterations in apoptotic pathways contribute to tumorigenesis and tumor progression, as they allow cancerous cells to survive longer, resist normally harmful stresses, and become more invasive. The expression of $A N X A 5$ is relatively high in node-positive bladder tumors (Mitra et al., 2006).

Connective tissue growth factor (CTGF) is a cysteine-rich secretory protein that can induce the proliferation, migration, and adhesion of and tube formation by vascular endothelial cells in culture. In addition, CTGF, in conjunction with other proteins, has been reported to contribute to bone metastasis, which can convert lowly metastatic breast cancer cells to highly metastatic cells. 
GTP-binding protein overexpressed in the skeletal muscle (GEM) belongs to the $\mathrm{RAD} / \mathrm{GEM}$ family of GTP-binding proteins. It is associated with the inner face of the plasma membrane and is thought to play a role in the regulation of receptor-mediated signal transduction (Maguire et al., 1994). Overexpression of mouse GEM is found to induce invasive pseudohyphal growth in Saccharomyces cerevisiae (Dorin et al., 1995). GEM is also found to revert the anchorage-independent growth and invasiveness of vascular endothelial zinc finger 1 transformed fibroblasts (Ward et al., 2002).

IL13RA1 is a subunit of the interleukin (IL)-13 receptor. This subunit forms a receptor complex with the IL-4 receptor alpha, a subunit shared by the IL-13 and IL-4 receptors (Obiri et al., 1997). This protein has been shown to bind tyrosine kinase (Hajoui et al., 2007), and thus may mediate the signaling pathways that lead to the activation of JAK1, STAT3, and STAT6 upon induction by IL-13 and IL-4. Activation of the JAK-STAT pathway can contribute to oncogenesis and tumor progression (Huang, 2007).

L-plastin (LCP1) is an actin-bundling protein that is abundantly expressed in hematopoietic cell lineages, and is overexpressed in many types of solid malignancies in humans. LCP1 is up-regulated in prostate cancer (Lin et al., 2000) and has been suggested to be a potential marker for metastasis in colorectal cancer (Otsuka et al., 2001). Bladder cancer tissue microarray analysis also indicates that LCP1 expression is significantly correlated with tumor grade (Harris et al., 2005, 2008). Prostatic acid phosphatase (ACPP) is an enzyme that catalyzes the conversion of orthophosphoric monoester to alcohol and orthophosphate. Its synthesis is regulated by androgen, and it is secreted by the epithelial cells of the prostate gland. In this study, we found that ACPP expression was up-regulated at the Ta-T1 transition but down-regulated at the T1-T2 transition. This difference may be attributed to the fact that advanced disease tends to be more anaplastic, thus causing the cells of advanced tumors to lose their capacity to synthesize this enzyme (Lange and Winfield, 1987).

Peroxiredoxins (PRDXs) are antioxidant enzymes that have an important role in cell differentiation, proliferation, and apoptosis. They are localized in the mitochondria. Enhanced PRDX I and VI expression is positively associated with a low rate of bladder cancer progression. Therefore, the down-regulation of the expression of PRDXs, such as PRDX3, may lead to cancer progression.

Because the expression of 1200 genes was found to be dysregulated at the Ta-T1 and Ta-T2 transitions, we further explored the pathways in which these genes may be involved. We identified 43 pathways, including cytokine-cytokine receptor interaction, the MAPK-signaling pathway, and the JAK-STAT-signaling pathway among others, to play an important role at the various stages of bladder cancer. These pathways have been demonstrated in previous studies (Nord et al., 2010) to be involved in tumor progression. For example, epidermal growth factor (EGF) is a potential mitogen for many different human tumors. Its effect is mediated via a bispecific receptor, the EGF receptor (EGFR), whose expression correlates well with invasive bladder cancer. The proteins interferon-gamma, tumor necrosis factor-alpha, and IL-1 $\alpha$ can decrease the expression of EGFR in some bladder tumor cell lines, thus inhibiting invasive tumor (Alexandroff et al., 1995). Toll-like receptor 4 (TLR4) and B7-H1, which are known from previous studies to be restricted to immune cells, are found to be aberrantly expressed in a bladder cancer, facilitating tumor evasion from immune surveillance and tumor progression. Activation of TLR4 signaling in bladder cancer cells up-regulated B7-H1 expression and this regulation is significantly attenuated by mitogen-activated protein kinase 1 (MAPK 1) and MAPK 8 inhibitors. In addition, the activation of TLR4 signaling results in the phosphoryla- 
tion of MAPK and PI3K pathway proteins and the up-regulation of IL-6 in a dose- and timedependent manner. All these proteins have been correlated with tumor grade in bladder cancer (Qian et al., 2009).

Furthermore, we focused our attention on the 38 genes identified as DEGs at the T1-T2 transition. Of these 38 genes, 13 genes also showed expression changes at the Ta-T1 transition, whereas 25 genes showed dysregulated expression exclusively at the T1-T2 transition. This suggests that these 25 genes may be crucial markers of the T1-T2 transition. We also explored the functions of these genes by KEGG pathway-enrichment analysis. We identified 2 important pathways through this analysis - ECM-receptor interaction and focal adhesion.

The interaction between tumor cells and ECM proteins, such as laminin, fibronectin, and collagen, plays a crucial role in tumor invasion and metastasis (Guiet et al., 2011). This interaction is facilitated through cell surface receptors such as integrins (Stipp, 2010). Therefore, laminin, fibronectin, collagen, and integrins may be involved in ECM-receptor interaction and the focal adhesion pathway.

As expected, we found the genes COL1A1, COL1A2, FN1, ITGA5, LGALS1, SPP1, VIM, POSTN, and COL18A1 to be up-regulated at the Ta-T2 and T1-T2 transitions. Collagen type I, the most abundant molecule within the collagen family, is a heterotrimer with $2 \alpha 1$ chains and $1 \alpha 2$ chain. This type of collagen represents the major fibrillar component of the stroma in most solid malignancies. In some aggressive tumors, collagen type I is overexpressed and plays a role in tumor development (Yang et al., 2007; Liang et al., 2008). Overexpression of FN1 has been found to correlate with a high histologic grade and an invasive phenotype (Kim et al., 2005). Niu et al. (2009) also identified differences in FN1 expression between superficial bladder transitional cell carcinoma and normal urothelial cells. They show that FN1 is up-regulated in tumor cells and is involved in the focal adhesion pathway. A correlation is also found between increased ITGA5 expression and histological stage. ITGA5 is positive in 6 (35.3\%) of the 17 invasive transitional cell carcinoma but in only $1(5.9 \%)$ of the 17 superficial transitional cell carcinoma. Galectin-1 is a protein that in humans is encoded by the LGALSI gene. Galectin-1 mRNA levels are highly increased in most high-grade tumors as compared with normal bladders or low-grade tumors (Cindolo et al., 1999). Memon et al. (2005) also reported that expression of galectin-1 increases in the later stages of bladder cancer. Constitutive expression of $O P N$ [also known as secreted phosphoprotein 1 (SPP1)] has been reported to be involved in the tumor carcinogenesis and metastasis of multiple human malignancies (Oates et al., 1997). SPP1 has been shown to be over-expressed in bladder cancer, and grade-3 tumors present higher SPP1 mRNA levels as compared to grade-2 tumors. Vimentin (VIM) is transcriptionally upregulated by $Z E B 2$ in tumors, and expression of VIM is confined to muscleinvasive disease. Periostin (POSTN) encodes a secreted $90-\mathrm{kDa}$ protein initially identified in a mouse osteoblastic library as a putative bone adhesion protein. Binding of POSTN to $\alpha \mathrm{V} \beta 3$ and $\alpha \mathrm{V} \beta 5$ integrins has been reported to promote cell adhesion and spreading. Higher POSTN expression levels are correlated with increased tumor aggressiveness and/or poorer survival in several malignant tumors (Tilman et al., 2007).

In conclusion, we have identified 36 genes that show differential expression in the transition of bladder cancer from the Ta to T1 stage and from the T1 to T2 stage. Therefore, these genes may serve as molecular markers for predicting tumor stage and progression. Eleven of these 36 genes are common to the Ta-T1 and T1-T2 transitions. However, they show expression changes in opposite directions at the 2 transitions. Twenty-five genes show 
differential expression exclusively at the T1-T2 transition. These genes may be involved in bladder cancer progression by affecting ECM-receptor interaction and focal adhesion. Importantly, cytokine-cytokine receptor interaction, neuroactive ligand-receptor interaction, the calcium-signaling pathway, the insulin-signaling pathway, the MAPK-signaling pathway, and the JAK-STAT-signaling pathway, among others, are associated with bladder cancer progression, irrespective of tumor stage.

\section{REFERENCES}

Alexandroff AB, Jackson AM, Chisholm GD and James K (1995). Cytokine modulation of epidermal growth factor receptor expression on bladder cancer cells is not a major contributor to the antitumour activity of cytokines. Eur. $J$. Cancer 31A: 2059-2066.

Askham JM, Platt F, Chambers PA, Snowden H, et al. (2010). AKT1 mutations in bladder cancer: identification of a novel oncogenic mutation that can co-operate with E17K. Oncogene 29: 150-155.

Benjamini Y and Hochberg Y (1995). Controlling the false discovery rate: a practical and powerful approach to multiple testing. J. R. Stat. Soc. Ser. B Stat. Methodol. 57: 289-300.

Burchill SA, Neal DE and Lunec J (1994). Frequency of H-ras mutations in human bladder cancer detected by direct sequencing. Br. J. Urol. 73: 516-521.

Castillo-Martin M, Domingo-Domenech J, Karni-Schmidt O, Matos T, et al. (2010). Molecular pathways of urothelial development and bladder tumorigenesis. Urol. Oncol. 28: 401-408.

Cindolo L, Benvenuto G, Salvatore P, Pero R, et al. (1999). Galectin-1 and galectin-3 expression in human bladder transitional-cell carcinomas. Int. J. Cancer 84: 39-43.

Dorin D, Cohen L, Del Villar K, Poullet P, et al. (1995). Kir, a novel Ras-family G-protein, induces invasive pseudohyphal growth in Saccharomyces cerevisiae. Oncogene 11: 2267-2271.

Guiet R, Van Goethem E, Cougoule C, Balor S, et al. (2011). The process of macrophage migration promotes matrix metalloproteinase-independent invasion by tumor cells. J. Immunol. 187: 3806-3814.

Hajoui O, Zheng H, Guay J, Letuve S, et al. (2007). Regulation of IL-13 receptor alpha 1 expression and signaling on human tonsillar B-lymphocyte subsets. J. Allergy Clin. Immunol. 120: 1425-1432.

Han KS, Jeong IG, Joung JY, Yang SO, et al. (2008). Clinical value of PTEN in patients with superficial bladder cancer. Urol. Int. 80: 264-269.

Harris LD, Tuziak T, De La Cerda J, Rosen D, et al. (2005). Tissue microarray analysis of pAkt, PTEN and L-plastin in urinary bladder cancer. Proc. Am. Assoc. Cancer Res. 46: 1314.

Harris LD, De La Cerda J, Tuziak T, Rosen D, et al. (2008). Analysis of the expression of biomarkers in urinary bladder cancer using a tissue microarray. Mol. Carcinog. 47: 678-685.

Hornigold N, Devlin J, Davies AM, Aveyard JS, et al. (1999). Mutation of the 9q34 gene TSC1 in sporadic bladder cancer. Oncogene 18: 2657-2661.

Huang S (2007). Regulation of metastases by signal transducer and activator of transcription 3 signaling pathway: clinical implications. Clin. Cancer Res. 13: 1362-1366.

Kim JH, Tuziak T, Hu L, Wang Z, et al. (2005). Alterations in transcription clusters underlie development of bladder cancer along papillary and nonpapillary pathways. Lab. Invest. 85: 532-549.

Kirkali Z, Chan T, Manoharan M, Algaba F, et al. (2005). Bladder cancer: epidemiology, staging and grading, and diagnosis. Urology 66: 4-34.

Lange PH and Winfield HN (1987). Biological markers in urologic cancer. Cancer 60: 464-472.

Li C, Li X, Miao Y, Wang Q, et al. (2009). SubpathwayMiner: a software package for flexible identification of pathways. Nucleic Acids Res. 37: e131.

Liang Y, Diehn M, Bollen AW, Israel MA, et al. (2008). Type I collagen is overexpressed in medulloblastoma as a component of tumor microenvironment. J. Neurooncol. 86: 133-141.

Lin CS, Lau A, Yeh CC, Chang CH, et al. (2000). Upregulation of L-plastin gene by testosterone in breast and prostate cancer cells: identification of three cooperative androgen receptor-binding sequences. DNA Cell Biol. 19: 1-7.

López-Knowles E, Hernandez S, Malats N, Kogevinas M, et al. (2006). PIK3CA mutations are an early genetic alteration associated with FGFR3 mutations in superficial papillary bladder tumors. Cancer Res. 66: 7401-7404.

Louhelainen JP, Hurst CD, Pitt E, Nishiyama H, et al. (2006). DBC1 re-expression alters the expression of multiple components of the plasminogen pathway. Oncogene 25: 2409-2419.

Maguire J, Santoro T, Jensen P, Siebenlist U, et al. (1994). Gem: an induced, immediate early protein belonging to the Ras 
family. Science 265: 241-244.

McGarvey TW, Maruta Y, Tomaszewski JE, Linnenbach AJ, et al. (1998). PTCH gene mutations in invasive transitional cell carcinoma of the bladder. Oncogene 17: 1167-1172.

Memon AA, Chang JW, Oh BR and Yoo YJ (2005). Identification of differentially expressed proteins during human urinary bladder cancer progression. Cancer Detect. Prev. 29: 249-255.

Mitra AP, Almal AA, George B, Fry DW, et al. (2006). The use of genetic programming in the analysis of quantitative gene expression profiles for identification of nodal status in bladder cancer. BMC Cancer 6: 159.

Niu HT, Zhang YB, Jiang HP, Cheng B, et al. (2009). Differences in shotgun protein expression profile between superficial bladder transitional cell carcinoma and normal urothelium. Urol. Oncol. 27: 400-406.

Nord H, Segersten U, Sandgren J, Wester K, et al. (2010). Focal amplifications are associated with high grade and recurrences in stage Ta bladder carcinoma. Int. J. Cancer 126: 1390-1402.

Oates AJ, Barraclough R and Rudland PS (1997). The role of osteopontin in tumorigenesis and metastasis. Invas. Metast. 17: $1-15$.

Obiri NI, Leland P, Murata T, Debinski W, et al. (1997). The IL-13 receptor structure differs on various cell types and may share more than one component with IL-4 receptor. J. Immunol. 158: 756-764.

Orlow I, LaRue H, Osman I, Lacombe L, et al. (1999). Deletions of the INK4A gene in superficial bladder tumors. Association with recurrence. Am. J. Pathol. 155: 105-113.

Otsuka M, Kato M, Yoshikawa T, Chen H, et al. (2001). Differential expression of the L-plastin gene in human colorectal cancer progression and metastasis. Biochem. Biophys. Res. Commun. 289: 876-881.

Qian Y, Deng J, Xie H, Geng L, et al. (2009). Regulation of TLR4-induced IL-6 response in bladder cancer cells by opposing actions of MAPK and PI3K signaling. J. Cancer Res. Clin. Oncol. 135: 379-386.

Ramírez-Backhaus M, Dominguez-Escrig J, Collado A, Rubio-Briones J, et al. (2012). Restaging transurethral resection of bladder tumor for high-risk stage Ta and T1 bladder cancer. Curr. Urol. Rep. 13: 109-114.

Sanchez-Carbayo M, Socci ND, Kirchoff T, Erill N, et al. (2007). A polymorphism in HDM2 (SNP309) associates with early onset in superficial tumors, TP53 mutations, and poor outcome in invasive bladder cancer. Clin. Cancer Res. 13: 3215-3220.

Stipp CS (2010). Laminin-binding integrins and their tetraspanin partners as potential antimetastatic targets. Expert. Rev. Mol. Med. 12: e3.

Tilman G, Mattiussi M, Brasseur F, van Baren N, et al. (2007). Human periostin gene expression in normal tissues, tumors and melanoma: evidences for periostin production by both stromal and melanoma cells. Mol. Cancer 6: 80.

van Rhijn BW, Lurkin I, Radvanyi F, Kirkels WJ, et al. (2001). The fibroblast growth factor receptor 3 (FGFR3) mutation is a strong indicator of superficial bladder cancer with low recurrence rate. Cancer Res. 61: 1265-1268.

Ward Y, Yap SF, Ravichandran V, Matsumura F, et al. (2002). The GTP binding proteins Gem and Rad are negative regulators of the Rho-Rho kinase pathway. J. Cell Biol. 157: 291-302.

Yang S, Shin J, Park KH, Jeung HC, et al. (2007). Molecular basis of the differences between normal and tumor tissues of gastric cancer. Biochim. Biophys. Acta 1772: 1033-1040. 